\title{
Kernos
}

Revue internationale et pluridisciplinaire de religion grecque antique

23 | 2010

Varia

\section{Hommage à François Jouan}

\section{Jocelyne Peigney}

Édition électronique
URL : http://journals.openedition.org/kernos/1556

DOI : 10.4000/kernos.1556

ISSN : 2034-7871

\section{Éditeur}

Centre international d'étude de la religion grecque antique

\section{Édition imprimée}

Date de publication : 1 janvier 2010

Pagination : 9-11

ISSN : 0776-3824

Référence électronique

Jocelyne Peigney, « Hommage à François Jouan », Kernos [En ligne], 23 | 2010, mis en ligne le 09 septembre 2011, consulté le 02 mai 2019. URL : http://journals.openedition.org/kernos/1556

Ce document a été généré automatiquement le 2 mai 2019.

Kernos 


\title{
Hommage à François Jouan
}

\author{
Jocelyne Peigney
}

1 Le $1^{\text {er }}$ juillet 2008, à l'Université de Paris 10, devant une assemblée que n'avaient découragée ni la date tardive dans l'année universitaire ni la chaleur d'un début d'été accablant, François Jouan recevait le volume de Mélanges qui lui était offert, Phileuripidès. Tout à une joie qu'il avait l'humour de mettre en scène dans ce lieu longtemps familier où il découvrait les derniers changements, il fit un discours plein d'émotion, de souvenirs et de clins d'œil, comme à son habitude. Il s'est éteint quelques mois plus tard, dans sa quatre-vingt-neuvième année, le 14 avril 2009, et c'est l'image souriante de l'été précédent que beaucoup garderont de lui.

2 Philologue, éditeur et commentateur d'Euripide, disciple de Louis Séchan, Fernand Chapouthier, Jean Humbert, Albert Severyns et Alphonse Dain, François Jouan était aussi un spécialiste de la mythologie grecque. Sa thèse principale porte sur Euripide et les légendes des Chants Cypriens. Des origines de la guerre de Troie à l'Iliade; parue en 1966 aux Belles Lettres, elle a été récompensée par le prix Zographos de l'Association des Études Grecques; sa thèse complémentaire intitulée Dion Chrysostome. Discours Troyen (XI) : Qu'Ilion n'a pas été prise, édition commentée inédite, l'avait emmené jusqu'à la rhétorique impériale sans le détourner du cycle troyen.

3 Outre la seconde édition en 1997 d'Euripide II, Hippolyte, Andromaque, Hécube, publié par Louis Méridier (1927), on lui doit six des douze volumes d'Euripide parus dans la Collection des Universités de France aux Belles Lettres : Euripide, VII, 1, Iphigénie à Aulis, édition parue en 1983, revue en 1990; les Fragments d'Euripide, parus de 1998 à 2003 (Euripide VIII, 1-4), édités en collaboration avec Herman Van Looy; Euripide, VII, 2, Rhésos , paru en 2004. À ces volumes s'ajoute l'édition du second tome des Tragiques grecs (t. 1 Eschyle, Sophocle; t. 2 Euripide) publiés en 2001 sous la direction de Bernard Deforge et de François Jouan dans la collection Bouquins, aux Éditions Robert Laffont.

Directeur après Francis Vian du Centre de Recherches Mythologiques de l'Université de Paris X, François Jouan a édité ou co-édité les Actes de quatre des rencontres trisannuelles organisées dans ce cadre: Visages du destin dans les mythologies, Actes du colloque de Chantilly, $1^{\mathrm{er}}-2$ mai 1980, Mélanges Jacqueline Duchemin, Paris, Les Belles Lettres, 1983; 
Mort et fécondité dans les mythologies, Actes du colloque de Poitiers, 13-14 mai 1983, Paris, Les Belles Lettres, 1986; Peuples et pays mythiques, Actes du Ve colloque du Centre de Recherches Mythologiques de l'Université Paris X, Chantilly, 18-20 septembre 1986, Paris, Les Belles Lettres, 1988, avec Bernard Deforge; Mythe et politique, Actes du colloque de Liège organisé par le Centre de Recherches Mythologiques de l'Université Paris X et le Centre d'Histoire des Religions de l'Université de Liège, 14-16 septembre 1989, Liège, 1990, avec André Motte. Il est l'auteur de plus de cent articles qui traitent du théâtre antique, particulièrement des mythes et de leur utilisation dans la tragédie, de la poésie lyrique, de l'histoire des textes ou de la réception de la littérature grecque classique jusqu'à l'époque contemporaine, mais aussi d'histoire des religions - il a participé au programme bibliographique Mentor de l'Université de Liège.

En employant toutes les ressources de l'érudition et des études techniques, François Jouan s'est intéressé jusqu'à la fin de sa vie à la reconstitution des drames perdus d'Euripide, mais il a eu en même temps la volonté constante de dégager dans les œuvres conservées les ressorts d'une création poétique rendue manifeste par la confrontation de la tradition épique et de la tragédie, indissociable du contexte intellectuel et historique de la deuxième moitié $d u v^{e}$ siècle. C'est là l'une des richesses de son livre, Euripide et les légendes des Chants Cypriens, réédité en 2009; c'est l'une des richesses des analyses qu'il a consacrées à la présentation des héros épiques mis sur la scène tragique, aux figures divines dans le théâtre attique, à la rhétorique chez Euripide, aux échos parodiques qui lient les œuvres entre elles ou à la paratragédie. Il a fait ainsi dialoguer les genres, les auteurs, les époques et mis sans cesse en regard les textes et les représentations figurées. Attentif à l'étude des interprétations antiques autant qu'il était ouvert aux différentes approches modernes, François Jouan a eu d'abord le souci des textes.

6 L'édition du Rhésos, si l'on veut s'en tenir aux derniers volumes publiés dans la CUF, en donne un autre exemple. La Notice fait un point des arguments avancés pour ou contre l'authenticité du drame, puis son auteur donne son sentiment personnel, contre l'attribution à Euripide - un sentiment nourri par la considération de « facteurs de choix fondés sur la sensibilité littéraire, appuyés sur une série de constatations objectives »-, pour conclure : « ... au fond là n'est pas l'essentiel : l'œuvre existe; si elle a des défauts, elle comporte des mérites qui lui sont propres, et c'est à les mettre en évidence, à tenter de dégager les influences subies par l'auteur et à la replacer dans la littérature vivante que la recherche philologique peut le mieux justifier son utilité » (p. XV-xvI).

7 Le compte rendu de Marco Fantuzzi dans la Bryn Mawr Classical Review en 2006 a souligné la qualité et l'intérêt de l'édition de François Jouan, parue quand la critique commençait à porter au drame une attention plus grande, mais en l'absence d'un commentaire récent publié. Après les travaux qui ont été menés à bien ces dernières années, on attend aujourd'hui deux commentaires du Rhésos, l'un préparé par Vayos Liapis, l'autre par Marco Fantuzzi précisément.

8 Quant à l'édition des Fragments d'Euripide, elle va bien au-delà du projet affiché de fournir une " édition facilement utilisable de [...] textes souvent mal connus », Marco Fantuzzi, recenseur élogieux encore, n'avait pas manqué de le dire. Le lecteur y trouve non seulement la traduction française d'un ensemble très vaste, mais aussi des notices érudites dotées d'une riche bibliographie qui permettent d'appréhender le traitement du mythe dans les différentes pièces, des hypothèses de reconstitution pour chaque œuvre perdue, tragédie ou drame satyrique, et des notes développées, comme il est d'usage désormais dans la CUF. L'édition de François Jouan et d'Herman Van Looy a reçu le prix 
Alfred Croiset de l'Académie des Inscriptions et Belles-Lettres et le prix Desrousseaux de l'Association des Études Grecques.

9 Helléniste de renom international, François Jouan s'est toujours engagé dans la vie de la recherche sans négliger ni les responsabilités administratives ni le devenir de l'enseignement des langues anciennes. Il a enseigné à Douai puis à Fès, comme jeune agrégé, à l'Université de Grenoble, comme assistant de Langues anciennes, puis à l'Institut français d'Athènes, comme professeur de Lettres, de 1951 à 1953; il fut ensuite à l'Université de Caen entre 1953 et 1977 assistant, maître-assistant, chargé d'enseignement, maître de conférences et professeur de grec, avant d'être professeur à l'Université de Paris X, où il était chargé de cours depuis 1974, jusqu'en 1989. Fondateur en 1965 du Collège Littéraire Universitaire du Mans qu'il dirigea pendant plusieurs années et dont est issue l'Université du Maine, directeur du CPR de Caen (Lettres) de 1970 à 1975, président de l'Association des Professeurs de Langues Anciennes de l'Enseignement Supérieur de 1972 à 1974, membre du Comité national du CNRS (1976-1982), membre du Conseil Supérieur des Universités (1983-1987), président de l'Association des Études grecques en 1981-1982, président de la Société française d'Histoire des Religions en 1982 et en 1989, responsable de la Formation de Recherches pluridisciplinaire de l'Université de Paris X « Image et cultures » (1984-1989), responsable du Groupe de Recherche Interuniversitaire sur la Tragédie grecque de 1984 à 1991, président de l'Association Mythe et Psychothérapie depuis 1991, directeur de la série grecque de la Collection des Études anciennes aux Belles Lettres jusqu'en 2006, il joua un rôle important dans la communauté universitaire.

François Jouan laisse une œuvre scientifique considérable, mais on ne peut évoquer ses travaux ou sa carrière sans évoquer les liens que ses activités lui avaient permis de nouer en Europe et bien au-delà; les contributions réunies dans le recueil Phileuripidès l'ont assez montré. Aux qualités humaines qui étaient les siennes et que tous rappellent, j'ajouterai le don qu'il avait de rendre courage à qui travaillait sous sa direction, en accordant sa confiance et son aide même quand les circonstances ou la lourdeur de la tâche poussaient à lâcher prise. Il a été un maître dont on n'oublie ni les conseils avisés ni la bienveillance, et qui faisait à ses disciples l'honneur de son amitié. Il laisse en chacun de ceux qui l'ont connu le souvenir d'un homme rigoureux, généreux et profondément bon. 